\title{
SPASME DE L'HÉMIFACE LA CHIRURGIE APPORTE UNE GUÉRISON DANS LA MAJORITÉ DES CAS
}

\author{
J. MAGNAN, A. MARDASSI, A. DEVEZE \\ SERVICE D'ORL HÔPITAL NORD MARSEILLE. FRANCE
}

\begin{abstract}
RESUME
Le spasme de l'hémiface est resté pour longtemps une maladie inconnue et énigmatique. De nos jours, la cause est clairement identifiée: un conflit vasculaire avec le nerf facial. L'imagerie par résonance magnétique permet le diagnostic en montrant l'artère responsable du conflit.

La chirurgie endoscopique de l'angle ponto-cérébelleux (APC) offre une localisation précise de la zone de conflit simplifiant ainsi l'approche chirurgicale.

Durant la période allant d'Octobre 1993 à Février 2010, on a opéré 662 patients souffrant de conflits neurovasculaires de I'APC, parmi eux 439 cas de spasme de l'hémiface. Tous les patients ont été opérés par voie rétrosigmoïde moyennant une endoscopie de l'APC et un monitoring du nerf facial.

À travers notre expérience, on discute les aspects pathogéniques, diagnostiques et thérapeutiques de cette affection.

Mots clés: spasme de l'hémiface, conflit neurovasculaire, imagerie par résonance magnétique, endoscopie de l'APC, voie rétrosigmoïde, monitoring du nerf facial.
\end{abstract}

\section{SUMMARY}

Hemifacial spasm was for a long time an unknown and enigmatic affection. Nowadays, the cause has been clearly identified: a vascular conflict with the facial nerve. Magnetic Resonance Imaging assesses the diagnosis showing the artery responsible of the conflict.

Endoscopic assisted surgery of the cerebellopontine angle (CPA) allows a precise localization of the conflict and simplifies the surgical procedure.

In the period between October 1993 to February 2010, we operated 662 patients suffering from CPA neurovascular conflict, among them 439 cases of hemifacial spasm. All patients were operated by retrosigmoid approach using CPA endoscopy and facial nerve monitoring.

Over our experience we discuss the pathogenic, diagnostic and therapeutic aspects of this affection.

Key words: hemifacial spasm, neurovascular conflict, magnetic resonance imaging, CPA endoscopy, retrosigmoid approach, facial nerve monitoring.

\section{INTRODUCTION}

Le spasme de l'hémiface est une entité anatomoclinique caractérisée par une symptomatologie évidente, de cause longtemps cachée et un conflit neurovasculaire à l'émergence du nerf facial $(1,2)$. L'imagerie par résonance magnétique en séquence CISS ${ }^{\circledR}$ (constructive interference in study state) est l'examen clé pour la mise en évidence de l'artère responsable. Par ordre de fréquence : artère cérébelleuse postérieure (ou Postero-InferiorCerebellar-Artery des anglosaxons : PICA), artère vertébrale, artère cérébelleuse moyenne (ou Antero-InferiorCerebellar-Artery : AICA) (3). Les techniques chirurgicales otoneurologiques modernes d'endoscopie et de monitoring du nerf facial apportent une grande fiabilité dans la localisation du conflit et une simplification de l'acte chirurgical $(1,4)$. Le spasme de l'hémiface est à la fois un symptôme et une maladie. Ces dernières années, un ensemble d'éléments nouveaux a permis de répondre à bon nombre d'interrogations concernant le spasme de
I'hémiface. Ainsi, le praticien peut avoir une attitude plus sereine vis-à-vis du malade, et une action curative vis-àvis de cette affection, éminemment invalidante sur les plans affectif et social (5).

\section{CLINIQUE}

Le spasme de l'hémiface est la contraction unilatérale, involontaire, brusque et isolée des muscles de la face (2). Il en résulte des grimaces asymétriques qui n'ont rien de commun avec la mimique volontaire (figure 1). Cette affection est plus fréquente chez la femme que chez I'homme dans une proportion de 4 pour 1. L'âge moyen révélateur est de 48 ans avec des extrêmes de 20 à 70 ans. Le spasme siège un peu plus souvent à gauche qu'à droite. Un élément déclenchant est quelquefois signalé par le patient : choc émotionnel, avulsion dentaire. 

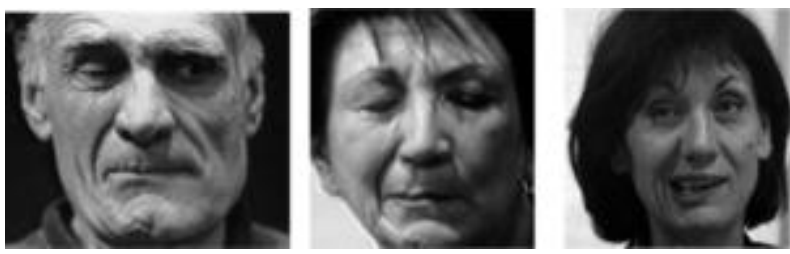

Fig. 1 : spasme de l'hémiface :

grimaces asymétriques unilatérales, involontaires, brusques et isolées.

La symptomatologie du spasme hémifacial présente quatre caractéristiques : unilatérale, involontaire, brusque et isolée.

Unilaterale : L'hémiface est plissée, l'œil à demi-clos, la pointe du nez incurvée, la commissure de la bouche est tirée en haut et en dehors, les fibres du peaucier se dessinent sous la peau. Le spasme n'a pas qu'un retentissement esthétique. II affecte aussi l'activité de l'individu, quand il perturbe la vision binoculaire, pouvant restreindre voire supprimer les possibilités de lecture ou de conduite automobile (6).

Brusque : Les contractions musculaires sont cloniques ou clonico-toniques, analogues à celles que provoquent les excitations électriques. Leur évolution est très caractéristique $(7,8)$.

- L'orbiculaire des paupières est le premier touché. C'est tout d'abord un frémissement de la paupière inférieure, puis des secousses fibrillaires, qui gagnent la paupière supérieure. Finalement une contraction spasmodique de l'orbiculaire détermine un blépharospasme unilatéral avec diminution de la fente palpébrale voire son occlusion complète (6).

- Ultérieurement, en quelques semaines, quelques mois, parfois même quelques années, le malade assiste à la diffusion progressive de l'atteinte vers la musculature de I'hémiface, qui devient alors le siège d'une grimace massive mettant en jeu tous les muscles.

- L'accès commence par des secousses cloniques qui partent de l'orbiculaire des paupières et gagnent de proche en proche, par bonds successifs les différents muscles. A son paroxysme, il atteint toute l'hémiface et persiste quelques secondes, créant l'asymétrie faciale caractéristique. La terminaison de cette phase tonique est le plus souvent brusque. Parfois, elle est suivie de quelques secousses cloniques.

La durée de l'accès est de quelques secondes à plus d'une minute. La répartition des crises est éminemment variable, mais le rythme a tendance à se rapprocher dans le temps. Parfois, les patients relatent une période de rémission spontanée de quelques mois, l'histoire naturelle allant vers l'augmentation en intensité et en fréquence (5). Isolée : Entre les accès, les intervalles sont complètement libres de tout trouble moteur : absence de contracture permanente et de déficit résiduel. En général, il n'y a aucun signe d'accompagnement. Le spasme de l'hémiface est isolée, sans phénomène douloureux, ni phénomène vasomoteur. L'audition et l'équilibre sont indemnes. Toutefois, il existe des formes cliniques rares associant spasme et troubles auditifs (VIII), spasme et otalgie réflexe (IX) ou spasme et hypertension artérielle (X).

\section{ETIOPATHOGENIE}

La notion de conflit neurovasculaire dans l'angle pontocérébelleux a été évoquée par Dandy (1935) à partir de constations opératoires chez des patients traités pour névralgie essentielle par rhizotomie rétrogasserienne (8). Cette hypothèse de boucle artérielle " offensante » au contact d'un nerf crânien a pu être affirmée lors d'une chirurgie pour spasme de l'hémiface par Gardner et Sava (1962) (8). Mais, c'est à Jannetta que revient la paternité de cette pathologie (6). II établit, aux cours des années 70 , à la fois les bases physiopathogéniques « des compressions pulsatiles » au niveau de la racine des nerfs crâniens et le concept chirurgical de décompression vasculaire (microvascular decompression, micro pour microscope opératoire) $(9,10)$. Toutefois pendant une vingtaine d'années, ce concept de compression ou conflit artèrenerf restera très controversé, Jannetta et ses élèves seront pour un temps relativement isolés. II faudra attendre les progrès de l'imagerie par résonance magnétique, le développement des techniques oto-neuro-chirurgicales moins invasives, les résultats présentés par les opérateurs et le témoignage des patients pour que progressivement le « mythe » devienne une « réalité » responsable au premier chef, des névralgies faciales et du spasme de l'hémiface.

Un certain nombre de causes organiques comprimant le nerf facial furent identifiées comme responsables du spasme de l'hémiface : anévrysme vertébro-basilaire, Cholestéatome de l'angle ponto-cérébelleux, syndrome d'Arnold Chiari, maladie de Paget...(8).

En dépit de ces cas rapportés dans la littérature, l'épine irritative agissant directement sur le nerf facial passait le plus souvent inaperçue lors de l'enquête étiologique. Histologiquement, l'idée d'une épine irritative sur le nerf facial a été évoquée par Babinski (11) qui parlait alors d'une « cicatrice tétanisante », pour bien marquer l'analogie entre le déroulement des phases du spasme de l'hémiface, et celle de la crise d'épilepsie ; l'épine irritative restant indéterminée quant à sa nature et à son origine sur le trajet du nerf facial. Ensuite, Gardner et al. mirent à l'honneur la notion de conflit vasculo-nerveux dans l'angle ponto-cérébelleux entre une boucle artérielle et la racine du nerf facial (12).

Un conflit neurovasculaire à l'émergence du nerf facial : Le vaisseau responsable induit une déformation des structures nerveuses dans $73 \%$ des cas (nerf facial et/ou fossette latérale du bulbe) le contact nociceptif et au niveau de la REZ du nerf facial dans $95 \%$ des cas, au 
niveau du porus dans $5 \%$ (8).

Les études expérimentales de neurophysiologie ont démontré les différents mécanismes producteurs d'influx nerveux lors d'une irritation chronique d'un nerf (figure 2). Au niveau de la zone de conflit, le contact artère-nerf crée d'une part un court-circuit axonal, et d'autre part l'émergence de foyers ectopiques d'activités, c'est-à-dire d'une synapse artificielle ectopique ou éphapse (théorie tronculaire éphaptique) $(8,13)$. L'éphapse joue le rôle d'épine irritative révélatrice d'une hyperexcitabilité endogène du noyau du VII (théorie nucléaire Moller et Janetta) (14). Ainsi, le vaisseau responsable crée une excitation ectopique, laquelle induit une stimulation chronique et des influx qui sont véhiculés de façon orthodromique vers la périphérie, et antidromique vers le noyau du nerf facial. Ce dernier hyperexcitable est hyperactivé, déclenchant une salve anormalement élevée de potentiel d'action orthodromique produisant l'accès de spasme (15).
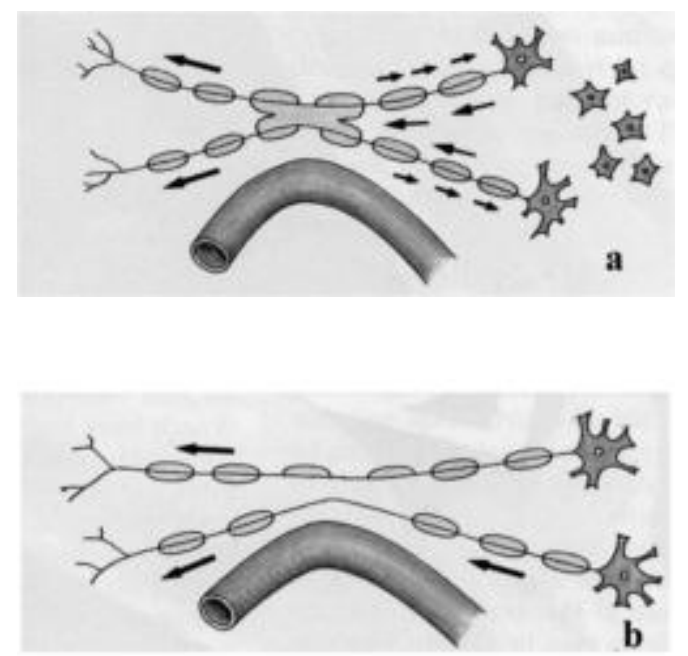

Figure 2 : Représentation schématique de la physiopathologie du conflit artério-nerveux :

a) Théorie électrostatique. b) Théorie éphaptique synapse ectopique diffusion transaxonale.

\section{DIAGNOSTIC}

L'incidence du spasme de l'hémiface, dit idiopathique par opposition au post paralytique, a été évaluée par Auger de 11 pour 100000 habitants (8).

D'octobre 1993 à février 2010, nous avons opérés 662 patients présentant un conflit neuro-vasculaire de l'angle pontocérébelleux, dont 439 cas de spasme de l'hémiface. Dans notre série, on a noté une prédominance féminine de 2 (290/439, 66\%) pour 1 homme (149/439, 33\%). L'atteinte est par définition unilatérale, et prédomine à gauche (251/439, 57\%). L'âge moyen révélateur est de 51 ans avec des extrêmes de 18 à 81 ans. Le délai moyen entre le début des signes et l'intervention est de 10 ans avec des extrêmes de 1 à 30 ans.

\section{Diagnostic positif :}

Le diagnostic de spasme de l'hémiface repose sur l'inspection et l'interrogatoire. L'examen électromyographique permet de mettre en évidence certaines anomalies qui semblent le caractériser (16).

- Sur l'étude en électromyographie conventionnelle (en stimulation-détection), on retrouve une réponse musculaire anormale retardée. Pour une stimulation sur la branche zygomatique du nerf facial, on enregistre au niveau du muscle orbiculaire des paupières et au niveau de muscles non innervés par cette branche zygomatique, une réponse musculaire décalée par rapport à la réponse directe de l'orbiculaire des paupières.

- En utilisant les épreuves de blink-reflex (ou trigéminofacial), on met en évidence un allongement de la latence de la première composante $\mathrm{R} 1 \mathrm{du}$ réflexe au niveau de l'orbiculaire des paupières, associé à une diffusion de cette composante au territoire inférieur (17).

Diagnostic différentiel :

II se pose avec les autres variétés de contractures de la face, mais en pratique il est aisément résolu $(5,18)$.

- Le spasme facial post-paralytique : se distingue par l'absence d'antécédents de paralysie faciale et l'absence de contracture permanente sur des muscles parésiés.

- Les tics convulsifs sont plutôt l'apanage de l'enfant (syndrome de Gilles de la Tourette), disparaissent pendant le sommeil et par le contrôle volontaire.

- Les dystonies neuromusculaires sont bilatérales (spasme facial médian de Meige ou paraspasme facial bilatéral de Sicard) et d'origine centrale.

- Les myokimies sont des contractions ondulantes de la paupière inférieure, non spastiques, évocatrices de sclérose en plaques (19).

\section{Diagnostic étiologique :}

II a pour but d'éliminer une cause tumorale et surtout de mettre en évidence la réalité du conflit vasculo-nerveux. A l'heure actuelle, l'imagerie par résonance magnétique représente la méthode la plus sensible et la moins invasive pour l'analyse des conflits vasculo-nerveux au niveau de l'angle ponto-cérébelleux.

L'IRM de l'angle ponto-cérébelleux en acquisition tridimensionelle en haute résolution est devenu l'examen princeps de tous les syndromes ou suspicion de syndrome de compression vasculaire des nerfs crâniens. Elle fait le diagnostic, évalue précisément le ou les vaisseaux responsables, permet un pronostic et une classification, et enfin pose l'indication opératoire $(3,9)$.

Les critères radiologiques principaux de conflit vasculonerveux dans l'angle ponto-cérébelleux sont dans tous les cas le lieu de conflit (REZ) et la déformation des structures nerveuses. La compression de la structure vasculaire orthogonale au trajet du nerf est un critère complémentaire, mais inconstant, notamment pour le nerf trijumeau ou lorsqu'il s'agit d'une veine (5). 
II faut demander une imagerie par résonance magnétique en séquence $\mathrm{CISS} \circledast$ (constructive interference in study state) laquelle fournit un excellent contraste entre les structures nerveuses, vasculaires et le liquide céphalorachidien grâce à son effet myélographique $(7,9,20)$ (figure 3,4$)$.

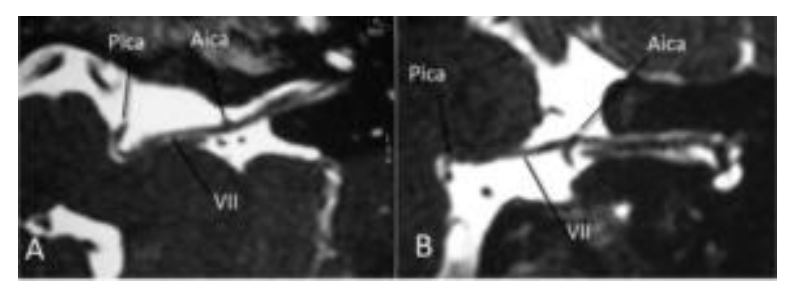

Fig.3 :

IRM en séquence CISS ${ }^{\circledR}$. Exemple d'un double conflit entre la PICA et l'émergence du nerf facial du côté gauche, avec un autre contact de l'AICA au niveau de la portion cisternale du nerf. $A$ : coupe transversale, $B$ : coupe frontale

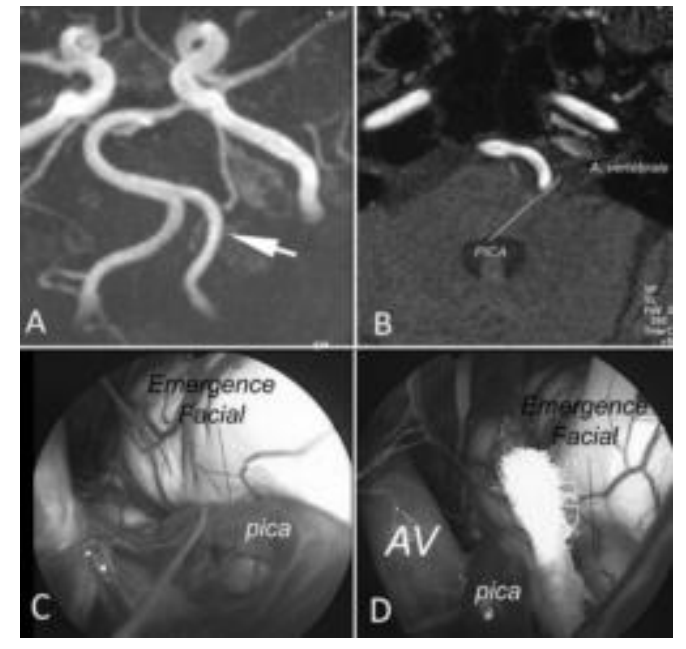

Fig. 4 : Démonstration radiologique de la malposition de l'artère vertébrale. L'artère vertébrale gauche est horizontalisée, amenant l'origine de la PICA au niveau de l'émergence du nerf facial.

A : IRM en séquence d'angio-IRM : artère vertébrale gauche horizontalisée (flèche), origine de la PICA au-dessus ;

B : coupe transversale: conflit au niveau de la fossette latérale du bulbe, avec impaction de l'artère vertébrale et de la PICA à l'émergence du nerf facial ;

C : vue endoscopique : PICA impactée dans la racine du nerf facial ; D : après décompression et interposition de téflon : empreinte laissée par la PICA sur la fossette latérale du bulble.

- Les artères les plus souvent en cause dans le spasme de l'hémiface sont l'artère cérébelleuse postéro-inférieure $(50 \%)$, puis l'artère vertébrale $(30 \%)$, et enfin, l'artère cérébelleuse antéro-inférieure $(20 \%)(7,21)$. II faut noter une participation polyvasculaire fréquente (22\%) du type artère vertébrale et artère cérébelleuse postéro-inférieu- re.

- La boucle artérielle induit une déformation ou une empreinte au niveau de la fossette latérale du bulbe dans $62 \%$ des cas (22).

\section{TRAITEMENT}

Traitement médical :

Il a toujours été décevant. La notion de «tics convulsifs 》 n'a pas reçu de confirmation thérapeutique en dépit de la large utilisation d'antiépileptiques (5). Le traitement par injection de toxine botulinique représente une récente et réelle avancée, même si son effet, purement symptomatique, n'est que palliatif et transitoire (23). La neurotoxine agit au niveau de la jonction neuro-musculaire entraînant une paralysie musculaire réversible. Les effets secondaires sont essentiellement dus à la diffusion vers les muscles voisins : ptosis, diplopie, paralysie faciale, toujours régressifs en 3 à 4 mois. Ainsi, l'injection de toxine botulinique nécessite d'être renouvelée tous les 3 à 4 mois, permettant ainsi d'avoir une amélioration clinique dans environ $80 \%$ des cas. Mais cet effet bénéfique tend à s'amenuiser dans le temps, responsable d'une certaine lassitude de la part du patient qui interrompt alors son traitement (7). Fait essentiel, la toxine botulinique effectuée préalablement à la chirurgie n'obère pas les résultats de cette dernière. Par contre, les alcoolisations et thermocoagulations du nerf facial sont à proscrire.

Traitement chirurgical :

II s'est clarifié et présente un regain d'intérêt. Toutes les interventions de neurolyse du nerf facial, quels qu'en soient le siège et le type, sont obsolètes. Seule demeure l'intervention de décompression vasculo-nerveuse prônée dès 1970 par Jannetta et al. (24). Controversée, pendant 20 ans,, cette opération a depuis reçu confirmation de ses bases étiopathogéniques (10). De plus, elle est devenue atraumatique grâce à la combinaison des améliorations successives des techniques otologiques, neurologiques et endoscopique ainsi que des progrès de l'anesthésie. Les points clés de la technique opératoire $(1,8,9,21,25)$ sont (figure 5) :

- L'abord rétromastoïdien limité $(15 \mathrm{~mm})$ pour seulement « effacer » le cervelet sans avoir recours à une rétraction instrumentale $(7,26)$;

- L'endoscopie de l'angle ponto-cérébelleux pour localiser avec exactitude le site du conflit artère-nerf sans dissection inutile $(4,6,27,28)$;

- Le monitoring du nerf facial pour contrôler l'efficacité de la décompression $(17,29,30)$;

- La mise en place d'une éponge de téflon qui joue le rôle « d'isolant électrique » entre le nerf facial et l'artère responsable déplacée (31) (figure 6). 

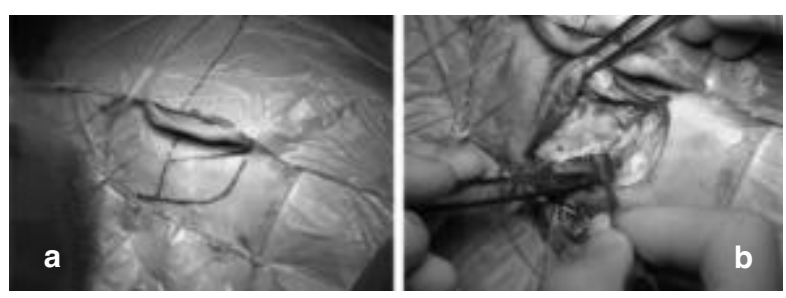

Figure 5 : Voie rétrosigmoïde

a) Repères cutanés.

b) Lambeaux cutanés et musculopériostés
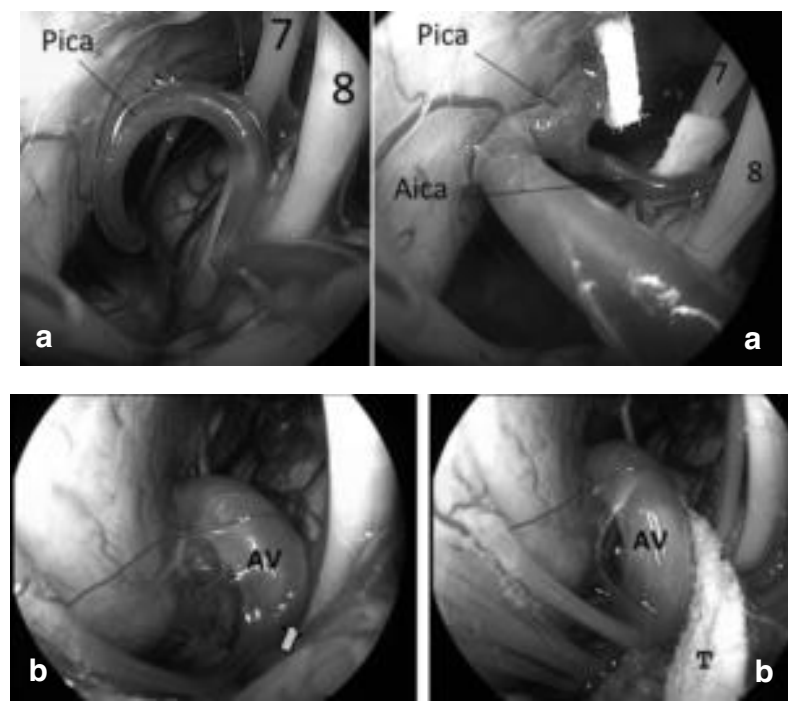

Fig. 6 : vue endoscopique de conflits artério-nerveux et interposition de téflon

a) Côté gauche. Spasme hémifacial du fait d'une artère Pica dominante, d'où provient l'Aica. A droite, la Pica entre en contact avec le nerf facial (7), qui est lui même comprimé par une pince artérielle du fait de l'origine de l'Aica à ce niveau. Après décompression, on visualise clairement l'Aica qui provient de la Pica. Le nerf facial est isolé par un fragment de téflon. La Pica est collée à la face postérieure du rocher, avec un téflon de sécurité.

b) conflit entre l'artère vertébrale (AV) et le nerf facial gauche (flèche) avant et après interposition de téflon $(\mathrm{T})$.

La durée de l'intervention est d'une heure et demie en moyenne, le patient regagne sa chambre le jour même, et est autorisé à sortir une semaine après (9). Nos résultats, chez 439 patients opérés, sont immédiats et durables dans $83 \%$ des cas, retardés de quelques semaines à 3 mois dans $9 \%$ des cas (figure 7 ). Les échecs (8\%) sont dus soit à une situation anatomique interdisant la mobilisation de l'artère, soit à un conflit vasculaire multiple.

Le risque pour le nerf facial est nul, celui pour la fonction auditive est devenu faible $3 \%(2,9,26)$. Ce n'est qu'une fois guéri que le patient raconte alors combien le spasme de l'hémiface est une affection invalidante et désespérante (18).

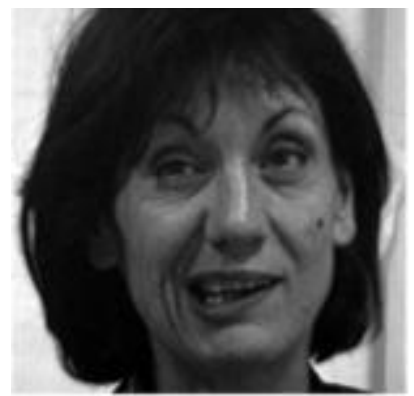

A

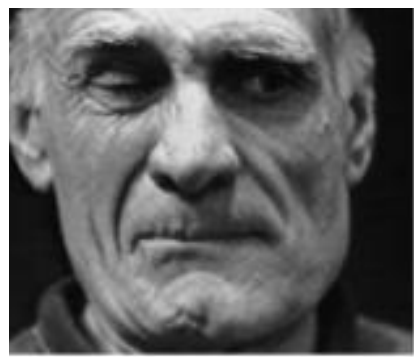

C

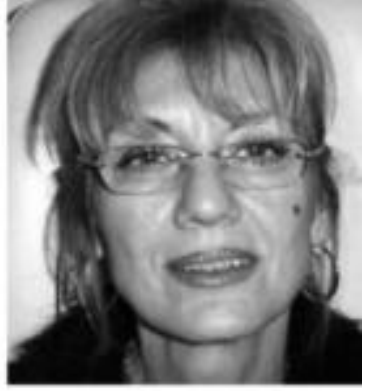

B

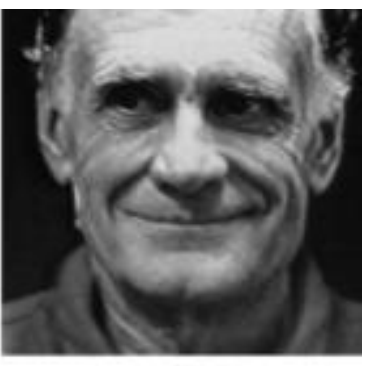

D
Figure 7 : Résultats thérapeutiques ( $A$ et $C$ : préopératoire, $B$ et $D$ postopératoire)

\section{CONCLUSION}

Le concept de conflit artère-nerf, proposé par Gardner et Jannetta pour expliquer et traiter le spasme de l'hémiface, a pendant longtemps été mis en doute, d'une part du fait de la présence à l'état normal de boucle artérielle asymptomatique au contact du paquet acoustico-facial et, d'autre part, de l'absence de documents objectifs faisant la preuve du contact neuro-vasculaire nociceptif. Grâce à I'IRM en préopératoire, à l'endoscopie en peropératoire et au monitoring électrophysiologique du nerf facial, le symptôme spasme de l'hémiface est devenu une entité anatomo-clinique bien définie dont le traitement logique est chirurgical. L'abord combiné microchirurgical et endoscopique de l'angle ponto-cérébelleux permet la suppression définitive de « la synapse artificielle » neurovasculaire avec un minimum de morbidité, même chez les patients âgés, dans $92 \%$ des cas. 


\section{REFERENCES}

1) Grotenhuis J A. The Use of the Endoscope in Microvascular Decompression Surgery. Operative Techniques in Neurosurgery 2001;4:162-5.

2) Huh R, Han I B, Moon J Y, Chang J W, Chung S S. Microvascular decompression for hemifacial spasm: analyses of operative complications in 1582 consecutive patients. Surgical Neurology 2008:69:153-7.

3) Ogiwara M, Shimizu T. Surface rendered three-dimensional MR imaging for the evaluation of trigeminal neuralgia and hemifacial spasm. Journal of Clinical Neuroscience 2004;11:840-4.

4) Nakaji P, Teo C. Endoscopic-Assisted Microvascular Decompression of the Cranial Nerves: Operative Technique. Oper Tech Neurosurg 2005;8:186-92.

5) Magnan J. Spasme de l'hémiface : La chirurgie apporte une guérison dans la majotité des cas. La Revue du Praticien $1994 ; 8: 27-32$.

6) Cheng W Y, Chao S C, Shen C C. Endoscopic microvascular decompression of the hemifacial spasm. Surgical Neurology 2008;70:40-6.

7) Sindou M, Keravel Y. Traitement neurochirurgical du spasme hémifacial primaire par décompression vasculaire microchirurgicale. Neurochirurgie $2009 ; 55$ :236-47.

8) Sauvain M O, Magistris M R, Tribolet N. Microvascular Decompression of the Facial Nerve. Operative Techniques in Neurosurgery 2001:4:127-36.

9) Magnan J, Caces F, Locatelli P, Chays A. Hemifacial spasm: Endoscopic vascular decompression. Otolaryngol Head Neck Surg 1997; 117:308-14.

10) Sindou M, Acevedo G. Microvascular Decompression of the Trigeminal Nerve. Operative Techniques in Neurosurgery 2001; 4:110-26

11) Babinski J. Hémispasme facial périphérique. Rev Neurol $1905 ; 13: 151-8$.

12) Gardner J, Sava G. Hemifacial spasm a reversible pathophysiologic state. J Neurosurg 1962;19:240-7.

13) Nielsen V. Pathophysiology of hemifacial spasm. Neurology 1984;34:41831.

14) Moller A, Jannetta P. Synkinesis in hemifacial spasm : results of recording intracranially from the facial nerve. Experientia 1985;41:415-7.

15) Wilkins R. Hemifacial spasmia review. Surg Neurol 1991;36:251-77.

16) Isch F, Jesel M. Hémispasme facial périphérique. Rev Prat (Paris) $1967 ; 17$ :3265-73.

17) Huang B R, Chang C N, Hsu J C. Intraoperative electrophysiological monitoring in microvascular decompression for hemifacial spasm. Journal of Clinical Neuroscience $2009 ; 16: 209-13$.

18) Kondo A, Tanabe H. What should be done, what should not be done in microvascular decompression surgery. International Congress Series 2004;1259:353-6

19) Jacobs $L$, Kaba $S$, Pullicino P. The lesion causing continuous facial myokymia in multiple sclerosis. Arch Neurol 1994;51:1115-9.

20) Borges A. Trigeminal neuralgia and facial nerve paralysis Eur Radiol 2005;15: 511-33.

21) Kurokawa Y, Maeda Y, Toyooka T, Inaba K. Microvascular decompression for hemifacial spasm caused by the vertebral artery: a simple and effective transposition method using surgical glue. Surg Neurol 2004;61:398-403.

22) Tallon Y. Exploration par IRM 3DFT des conflits vasculo-nerveux au niveau de l'angle ponto-cérébelleux : spasme de l'hémiface et névralgie faciale. Thèse Med Univ Bordeaux II, Marseille II $1994 ; n^{\circ} 3021$.

23) Elbaz D. Traitement du blépharospasme et de l'hémispasme facial par la toxine botulinique. Ann Oto Laryngol (Paris) 1994 ;111:141-4.

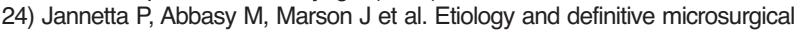
treatment of hemifacial spasm. J Neurosurg 1977;47:321-8.

25) Magnan J, Chays A, Caces F et al. Place de l'endoscopie et de la décompression vasculaire dans le traitement du spasme de l'hémiface. Ann Otolaryngol Chir Cervicofac $1994 ; 111: 153-60$.

26) Lovely TJ, Getch CC, Jannetta PJ. Delayed facial weakness after microvascular decompression of cranial nerve VII. Surg Neurol 1998;50:449-52. 27) Chen M J, Zhang W J, Yang C, WU Y Q, Zhang Z Y, Wang Y. Endoscopic neurovascular perspective in microvascular decompression of trigeminal neuralgia. Journal of Cranio-Maxillofacial Surgery 2008;36:456-61.

28) Jarrahy R, Berci G, Shahinian H K. Endoscope-assisted microvascular decompression of the trigeminal nerve. Otolaryngol Head Neck Surg 2000;123:218-23.

29) Fukuda M, Oishi M, Fujii Y. Facial nerve motor evoked potential monitoring during microvascular decompression for hemifacial spasm. Society Proceedings in Clinical Neurophysiology 2009;120:147-80.

30) Joo W I, Lee K J, Park H K, Chough C K, Rha HK. Prognostic value of intraoperative lateral spread response monitoring during microvascular decompression in patients with hemifacial spasm. Journal of Clinical Neuroscience 2008;15:1335-9.

31) Chen J-F, Lee S-T, Lui T-N, Yeh Y-S, Chen T-Y, Tzaan W-C. Teflon granuloma after microvascular decompression for trigeminal neurologia. Surg Neurol 2000;53:281-7. 
